\title{
ANÁLISE COMPARATIVA DA MOBILIDADE FUNCIONAL E MEDO DE QUEDAS DE IDOSAS COMUNITÁRIAS
}

\section{COMPARATIVE ANALYSIS OF FUNCTIONAL MOBILITY AND FEAR OF ELDERLY FALLS COMMUNITY}

\author{
Daniela Virote Kassick Müller ${ }^{\text {a }}$ \\ Orcid: https://orcid.org/0000-0003-1800-3966
}

Jéssica Sanchotene de Bastos ${ }^{\mathbf{b}^{* *}}$
Orcid: https://orcid.org/0000-0003-0105-7204

danivkm@gmail.comª je.sbastos@hotmail.com ${ }^{\mathrm{b}}$

Universidade Federal do Pampa - Unipampa, Campus Uruguaiana - RS*, Núcleo de Apoio à Saúde da Família da Prefeitura Municipal de Barra do Quaraí - RS ${ }^{* *}$

\section{RESUMO}

Data de Submissão :20/08/2019 Data de Aceite: 21/10/2019

Introdução: A população mundial está envelhecendo e, com isso, é possível ocorrerem diversas modificações estruturais e fisiológicas no organismo que podem levá-lo ao déficit de equilíbrio e quedas. Tais quedas podem trazer tanto consequências físicas quanto psicológicas, portanto a importância de preveni-las. Objetivo: Comparar a mobilidade funcional de idosas com e sem medo de cair. Materiais e Métodos: Trata-se de um estudo comparativo, realizado com

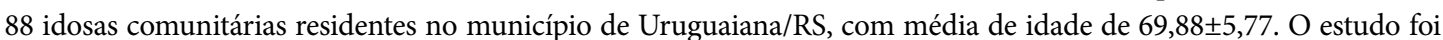
submetido e aprovado pelo Comitê de Ética e Pesquisa da Universidade Federal do Pampa (CEP-UNIPAMPA), de acordo com pareceres número 930.945/15 e 1.743.674/16. Foram realizadas avaliações seguindo o seguinte protocolo: a) aplicação de questionário sobre o perfil sociodemográfico, nível de atividade física, histórico de quedas e outras alterações associadas, tais como alterações visuais, auditivas e labirínticas; b) investigação sobre a preocupação em sofrer quedas (FES-I); c) mobilidade funcional (Timed Up and Go - TUG e velocidade de marcha). A partir da aplicação do FES-I, as idosas foram divididas em dois grupos de acordo com sua classificação: idosas sem medo de cair (grupo 1= 48 idosas $(54,5 \%)$ ) e o grupo 2 pelas idosas com medo de cair (40 idosas - 45,5\%). Os resultados demonstraram que a média do TUG entre as idosas do grupo 1 foi menor $(\mathrm{p}=0,005)$, assim como a velocidade da marcha foi maior entre estas idosas ( $\mathrm{p}=0,006)$. Resultados e Conclusões: Sendo assim, o estudo demonstrou que aquelas idosas que apresentam maior medo de cair apresentam piores resultados em sua mobilidade funcional quando comparadas a idosas com menor preocupação com quedas.

Palavras-chave: Acidentes por quedas; equilíbrio postural; envelhecimento; limitação da mobilidade

\section{ABSTRACT}

Introduction: The world population is aging and, as a result, several structural and physiological changes can occur in the body that can lead to balance deficit and falls. Such falls can have both physical and psychological consequences, so the importance of preventing them. Objective: To compare the functional mobility of elderly women with and without fear of falling. Materials and Methods: This is a comparative study conducted with 88 community-dwelling elderly women living in the municipality of Uruguaiana / RS, with a mean age of $69.88 \pm 5.77$. The study was submitted and approved by the Ethics and Research Committee of the Federal University of Pampa (CEP-UNIPAMPA), according to numbers 930,945 / 15 and 1,743,674 / 16. Assessments were performed according to the following protocol: a) application of a questionnaire on socio-demographic profile, level of physical activity, history of falls and other associated changes, such as visual, auditory and labyrinthine alterations; b) investigation into the concern about suffering falls (FES-I); c) functional mobility (Timed Up and Go - TUG and walking speed). From the application of FES-I, the elderly were divided into two groups according to their classification: elderly without fear of falling (group $1=48$ elderly (54.5\%)) and group 2 by the elderly with fear of falling. ( 40 elderly $-45.5 \%$ ). The results showed that the average TUG among the elderly women in group 1 was lower $(\mathrm{p}=0.005)$, as well as the gait speed was higher among these elderly women $(\mathrm{p}=$ 0.006). Results and Conclusions: Thus, the study showed that older women who are more afraid of falling have worse results in their functional mobility when compared to older women who are less concerned about falls.

Keywords: Accidental Falls, Postural Balance, Aging, Mobility Limitation. 


\section{Introdução}

O envelhecimento da população mundial é um evento natural que vem ocorrendo de forma rápida e progressiva, principalmente nos países em desenvolvimento. No Brasil, além do crescimento da população idosa, observa-se um importante aumento na expectativa de vida, o que contribui de forma significativa para o processo de transição demográfica em que o país se encontra ${ }^{1,2}$.

Com o avanço da idade, ocorrem diversas modificações estruturais e fisiológicas nos sistemas de integração sensorial e neuromuscular do nosso corpo. Entre as alterações do sistema locomotor, podemos destacar a redução da flexibilidade, da função e da massa muscular, da mobilidade e rigidez articular, déficits na coordenação motora, equilíbrio e instabilidade postural ${ }^{3}$. Essas mudanças, associadas às doenças crônicas e fatores externos, podem comprometer a marcha e a mobilidade funcional da pessoa idosa, tornando-a mais propensa a quedas ${ }^{4}$.

As quedas afetam cerca de $30 \%$ dos idosos ao ano, sendo um fator muito preocupante nessa população, devido às suas consequências físicas e psicológicas como ansiedade, depressão, isolamento social, fraturas, dependência física, hospitalização, institucionalização, restrição ao leito, e até mesmo morte ${ }^{5}$.

A avaliação do sentimento de medo em relação às quedas não é comum na prática clínica; porém, pode ajudar o fisioterapeuta a determinar o motivo dessa condição e contribuir na elaboração de estratégias de prevenção e tratamento, para diminuir as limitações na vida do idoso ${ }^{6}$.

A utilização de testes físicos, funcionais e de equilíbrio também é interessante durante a avaliação da saúde geral para determinar o perfil funcional do idoso, investigar quais as funções que podem estar comprometidas e identificar situações de risco potencial para quedas ${ }^{7}$.

A preocupação em cair está associada à redução de mobilidade e atividade física. Porém, ainda existem poucos estudos na literatura que investigam a influência do medo de cair na mobilidade e equilíbrio do idoso, através de questionários associados a testes funcionais. Acredita-se que idosos com medo de cair apresentem um menor desempenho da mobilidade funcional e equilíbrio, devido à falta de segurança em realizar suas atividades. Sendo assim, o estudo tem como objetivo comparar a mobilidade funcional de idosas com e sem medo de cair.

\section{Casuística e métodos}

O presente estudo faz parte de uma pesquisa maior, sendo submetido e aprovado pelo Comitê de Ética e Pesquisa da Universidade Federal do Pampa (CEP-UNIPAMPA), de acordo com pareceres número 930.945/15 e 1.743.674/16.

Trata-se de um estudo comparativo, realizado com idosas comunitárias residentes no município de Uruguaiana/RS, as quais atenderam aos seguintes critérios de inclusão: voluntárias com idade igual ou superior a 60 anos, capacidade cognitiva preservada, independência funcional da marcha, com ou sem uso de dispositivos auxiliares e liberação médica para prática de exercícios físicos. Não foram incluídas no estudo aquelas que apresentaram histórico de doenças neurológicas, motoras, cognitivas ou psiquiátricas, qualquer nível de amputação de membros inferiores, anquilose articular de membros inferiores e hipertensão arterial não controlada durante aquisição dos dados.

As avaliações foram realizadas na Universidade Federal do Pampa (UNIPAMPA), campus Uruguaiana, cidade de Uruguaiana, RS, em dia e horário pré-determinados, nos períodos de fevereiro de 2015 a maio de 2017. As avaliações seguiram o seguinte protocolo. Inicialmente foi aplicado um questionário estruturado, contendo os dados de identificação do sujeito, perfil sociodemográfico, informações sobre o nível de atividade física, histórico de quedas e outras alterações associadas, tais como alterações visuais, auditivas e labirínticas. Além disso, foi investigada a preocupação das idosas em sofrer quedas e a mobilidade funcional. Para a avaliação destas variáveis foram utilizados os seguintes instrumentos de pesquisa:

1) Falls Efficacy Scale-International (FESI-BRASIL): questionário composto por 16 questões, que determina o nível de preocupação ao realizar atividades da vida diária. A pontuação 
varia de 1 a 4 , sendo 1 ausência de preocupação e 4 extremamente preocupado em cair. A pontuação total pode variar de 16 a 64 pontos. Valores a partir de 26 referem-se à preocupação com quedas em relação às atividades diárias, sendo que valores inferiores demonstrariam ausência de preocupação $0^{6,8}$. A escala foi validada em 2010 para avaliar o medo de cair na população de idosos brasileiros da comunidade por Camargos et $\mathrm{al}^{9}$.

2) Teste Timed Up and Go (TUG): foi realizado para avaliar a mobilidade funcional, determinada pela velocidade que o idoso realiza a atividade de levantar de uma cadeira, andar três metros, dar uma volta de $180^{\circ}$ e retornar à posição inicial. Valores maiores ou iguais a 13,5 segundos indicam alta probabilidade de quedas e valores menores indicam idosos não caidores ${ }^{8}$. O teste foi traduzido para o português e validado por Dutra et al (2016), apresentando confiabilidade para aplicação em idosos brasileiros ${ }^{10}$.

3) Velocidade da Marcha (TVM6): O teste de velocidade de marcha de seis metros é considerado como uma medida rápida, de baixo custo e com boa reprodutibilidade em idosos da comunidade para predizer quedas. A avaliação da velocidade da marcha foi realizada solicitando ao idoso que caminhasse normalmente percorrendo uma distância de 6 metros. Foi registrado o tempo que o idoso realizou a tarefa e calculada a velocidade da marcha dividindo-se a distância pelo tempo. O tempo foi mensurado a partir do uso de um cronômetro digital ${ }^{11,12}$.

A fim de atender ao objetivo proposto pelo estudo, as idosas foram divididas em dois grupos de acordo com a classificação obtida pelo questionário FES-I-BRASIL entre idosas sem medo de cair (grupo 1) e o grupo 2 pelas idosas com medo de cair. Os resultados foram colocados em tabelas do Microsoft Excel e analisados com uso do pacote estatístico SPSS versão 17.0. Para análise das variáveis categóricas ou qualitativas nominais foi realizado o teste qui-quadrado e a comparação das variáveis quantitativas ou numéricas contínuas foi feita por meio do teste t-Student para amostras independentes. Para esse estudo, qualquer diferença ou correlação tal que $\mathrm{p}<0,05$ foi considerada estatisticamente significativa.

\section{Resultados}

A amostra foi composta de 88 voluntárias do sexo feminino, com idade a partir de 60 anos. A idade média foi de $69,88 \pm 5,77$. A partir do ponto de corte utilizado para classificação do medo de cair obteve-se, 48 idosas (54,5\%) não apresentando o sentimento de medo de quedas, enquanto 40 (45,5\%) apresentaram essa preocupação.

Num primeiro momento foi feita a relação através do teste qui-quadrado entre a presença do medo de cair, com resposta sim ou não no questionário com os resultados da classificação do FES-I através do seu ponto de corte, sendo demonstrada forte associação entre ambos com resultado estatisticamente significativo $\left(\mathrm{p}<0,0001^{\star}\right)$. Neste caso, foi observado que a maioria das idosas pertencentes ao grupo 1 pela FES-I (sem medo) relatou não ter medo de quedas $(62,5 \%)$ enquanto apenas $18(37,5 \%)$ referiram preocupação em cair. Já as idosas que compõem o grupo 2 pela FES-I (com medo) apresentaram relato de medo de cair em $90 \%$ dos casos.

Não houve resultados significativos na comparação entre as idosas com e sem medo de cair em relação ao seu histórico de quedas, número de quedas no último ano, histórico de fraturas e prática de atividade física regular. Além disso, não se observou relação entre o medo de cair com a presença de sintomas de síndrome vertiginosa (zumbido, tontura, sensação de flutuação da cabeça, vertigem); alterações visuais ou auditivas e uso de órteses corretivas para visão e/ou audição.

Os resultados da avaliação da mobilidade funcional entre os grupos 1 e 2 são apresentados na Tabela 1, abaixo. 
Tabela 1 - Resultados da avaliação de equilíbrio e mobilidade funcional entre idosos com e sem medo de quedas.

\begin{tabular}{|c|c|c|c|c|}
\hline & Divisão FES & Média & Desvio Padrão & $\mathbf{p}$ \\
\hline \multirow{2}{*}{ Média TUG (seg) } & Sem medo & 11,0668 & 2,2040 & \multirow{2}{*}{$0,005^{\star}$} \\
\hline & Com medo & 12,8223 & 3,4704 & \\
\hline \multirow{3}{*}{$\begin{array}{l}\text { Velocidade marcha } \\
\text { (m/seg) }\end{array}$} & Sem medo & 0,8317 & 0,2178 & \multirow{3}{*}{$0,006^{*}$} \\
\hline & Com medo & 0,7135 & 0,1715 & \\
\hline & Com medo & 127,8250 & 47,0405 & \\
\hline
\end{tabular}

* Teste $t$ de Student para amostras independentes.

Nela, é possível verificar que no TUG as idosas do grupo 1 (sem medo de queda) apresentaram uma média de 11,06 segundos, enquanto as do grupo 2 (com medo de cair) apresentaram valor de 12,82 segundos $(p=0,005)$. Fato que demonstra que as idosas sem medo foram mais rápidas na execução da tarefa.

Já no teste TVM6, as idosas pertencentes ao grupo 1 foram mais velozes que as com medo de cair, já que tiveram velocidade média de $0,83 \mathrm{~m} /$ seg. ao passo que as idosas do grupo 2 velocidade de $0,71 \mathrm{~m} / \mathrm{seg}$.

\section{Discussão}

Atualmente, no Brasil, observa-se um processo de transição demográfica, no qual vem ocorrendo um aumento do número de mulheres na população idosa. Hoje, as mulheres representam 55,5\% da população idosa brasileira e $61 \%$ de idosos acima de 80 anos. As idosas do sexo feminino, sobretudo, acima de 80 anos, apresentam piores condições de saúde, tornando-as mais dependentes de cuidados $^{13}$.

De acordo com a literatura, o medo de cair é uma das consequências de quedas anteriores; porém, também pode ocorrer em indivíduos que não caíram. O conceito de autoeficácia é a forma mais utilizada e reconhecida cientificamente para mensurar o medo de cair. Sendo assim, pode ser definida como o elemento cognitivo do medo de quedas. A preocupação em cair pode ser considerada um fator protetor, uma vez que este impede que o indivíduo entre em situações de risco. No entanto, este medo pode ser exagerado, interferindo na qualidade de vida, no conforto emocional e no desempenho do idoso, restringindo sua participação social e provocando isolamento social ${ }^{14}$.

Um estudo de Pinheiro, Vilaça e Carvalho $(2014)^{15}$ analisou a estabilidade postural, o risco de quedas e o medo de cair em idosos com neuropatia diabética (DNP) que realizam exercícios terapêuticos. Verificou-se que 20 a $60 \%$ dos pacientes experimentaram medo de cair sem ter ocorrido o episódio da queda. Além disso, a frequência foi maior nas mulheres, principalmente aquelas que são sedentárias ou têm histórico de depressão. Observa-se que até os 75 anos de idades as mulheres tendem a cair mais que os homens, sendo que as frequências se igualam entre os sexos após essa idade.

Acredita-se que a maior incidência de quedas em mulheres ocorre devido à redução de massa óssea e consequente aumento da disposição para osteoporose, à redução do estrógeno, diminuição da força muscular, à maior incidência de doenças crônicas, maior exposição a atividades domésticas e a comportamentos de riscos maiores quando comparado aos homens ${ }^{16}$.

$\begin{array}{ccr}\text { Vale ressaltar } & \text { que } & \text { existem } \\ \text { outras condições de } & \text { risco } & \text { que }\end{array}$ podem provocar um evento de queda. Elas podem ser multifatoriais e envolvem condições intrínsecas e extrínsecas. Entende-se por fatores intrínsecos aqueles decorrentes das alterações fisiológicas relacionadas ao envelhecimento. Os fatores extrínsecos, por sua vez, estão relacionados a ambientes inseguros, com barreiras, mal planejados e mal construídos, com presença de escadas, ausência de 
corrimãos, iluminação inadequada, tapetes soltos e obstáculos (fios elétricos, pisos malconservados, por exemplo) no local de circulação ${ }^{17}$.

A utilização do TUG e da velocidade da marcha para avaliar a mobilidade funcional de idosos vêm sendo amplamente relatada na literatura e é recomendada pela Sociedade Geriátrica Britânica e Americana para classificar o risco de quedas ${ }^{18}$. No presente estudo, verificou-se associação entre o medo de quedas e os resultados do TUG e da velocidade da marcha. Observou-se que idosos que não apresentam medo de cair, obtiveram melhor desemprenho nos testes funcionais em relação aos idosos sem medo.

Em uma análise realizada por Padoin e colaboradores, em $2010^{18}$, utilizando a variável medo de cair, 34 (62\%) dos sujeitos estudados referiram ter medo de cair. Na comparação entre o medo de cair e o TUG, o resultado foi menor nos sujeitos sem medo $(12,2$ segundos $\pm 3,4)$ do que naqueles com medo de cair $(17,1$ segundos \pm 10 ; $\mathrm{p}=0,045$ ), o que corrobora com os achados desta pesquisa. Sendo assim, pode-se afirmar que quanto menor o tempo de realização no TUG, melhor pode ser a condição de equilíbrio dinâmico ${ }^{19}$.

Neste estudo, a redução da velocidade de marcha em idosos foi associada a uma baixa autoeficácia para quedas; porém, alguns autores acreditam que não existe correlação entre a velocidade de marcha e o equilíbrio. Ou seja, não é possível afirmar que a redução da velocidade entre os idosos ocorra como uma estratégia compensatória para assegurar um equilíbrio adequado. Talvez este mecanismo ocorra entre aqueles que sofrem quedas recorrentes ${ }^{20}$. No entanto, Padoin et al (2010) ${ }^{18}$ referem que idosos com baixa velocidade de marcha apresentam maior risco de quedas, ou seja, quanto mais lenta for a marcha, maior a instabilidade postural.

Nunes et al. (2016) ${ }^{21}$ afirmam que os indivíduos ativos apresentam melhor equilíbrio corporal e menos medo de cair, indicando que o nível de atividade física parece ser um fator importante para diminuir a preocupação em cair, o que difere dos resultados do nosso estudo. Sendo assim, a atividade física tem sido sugerida como uma estratégia fundamental para a prevenção de quedas e melhora da qualidade de vida de idosos.
Uma das limitações deste estudo foi o fato de avaliar somente mulheres, em função de um número reduzido de idosos do sexo masculino no local de recrutamento dos voluntários. A amostra foi composta, em sua maioria, por idosas socialmente ativas devido à maior participação de mulheres nos grupos de convivência e na prática de atividade física. Essa característica pode ser verificada em muitos outros estudos, pois de acordo com a literatura, as mulheres apresentam maior limitação funcional e um declínio mais pronunciado de força muscular, que é um componente relacionado à velocidade de marcha ${ }^{16}$.

Outra variável não analisada foi o número de doenças apresentadas pelas idosas, o que pode influenciar nos resultados, pois idosos que apresentam alguma enfermidade são mais propensos às quedas, devido às alterações no equilíbrio e na estabilidade corporal ${ }^{21}$.

Sendo assim, além de promover diversos benefícios para a saúde e bem-estar geral do idoso, a atividade física é um fator determinante para um bom processo de envelhecimento, melhora da expectativa e qualidade de vida, assim como na reintegração do idoso na sociedade ${ }^{3}$.

Para evitar as quedas, além das abordagens terapêuticas, devem-se adotar medidas como a educação e o autocuidado, utilização de dispositivos auxiliares para a marcha (quando necessário), utilização criteriosa de medicamentos, evitando-se em especial, aqueles que podem causar hipotensão postural, e a adaptação do ambiente em que o indivíduo vive, principalmente dentro de casa. Desta forma, deve-se considerar o planejamento da casa para que a mesma esteja segura e funcional ${ }^{16}$.

Existem diversos programas de prevenção de quedas; entretanto, são poucos os que consideram o medo de cair como parte importante na avaliação dos idosos, devendo-se incrementar a atenção a este aspecto através de uma maior sensibilização por meio dos profissionais da saúde para que se busquem estratégias adequadas a esta população. Além de ser importante analisar fatores físicos e ambientais do contexto do idoso, deve-se levar em conta sintomas emocionais relacionados à queda, bem como se o idoso apresenta apoio social e familiar, fatores esses fundamentais para um tratamento bem sucedido ${ }^{14}$. 


\section{Conclusão}

O presente estudo demonstrou que idosas que apresentam medo de cair acabam desenvolvendo uma redução na sua mobilidade funcional, desempenhando suas atividades de forma mais lenta, provavelmente pelo próprio receio da queda.

Sendo assim, é preciso identificar aqueles indivíduos que necessitam de intervenção, direcionar o planejamento do tratamento de acordo com a necessidade do idoso, considerando suas preocupações e limitações físicas, além de verificar a eficácia das intervenções realizadas através da avaliação contínua do paciente.

Desta forma, sugere-se a realização de estudos posteriores que abranjam uma população maior, contemplando o sexo masculino, assim como ensaios clínicos que verifiquem a influência da intervenção fisioterapêutica na modificação do medo de quedas e na melhora da mobilidade e equilíbrio postural.

\section{Conflitos de interesse}

Os autores declaram que participaram da concepção, análise de resultados e contribuíram efetivamente na realização do artigo: "AVALIAÇÃO DO MEDO DE QUEDAS E SUA INFLUÊNCIA NA MOBILIDADE FUNCIONAL DE IDOSAS COMUNITÁRIAS”. Tornam pública a responsabilidade pelo seu conteúdo, que não foram omitidas quaisquer ligações ou acordos de financiamento entre os autores e companhias que possam ter interesse na publicação deste artigo. Afirmam que não tem qualquer conflito de interesse com o tema abordado no artigo, nem com os produtos/itens citados. Declaramos que o artigo citado acima é original e que o trabalho, não foi enviado a outro periódico científico e não o será, enquanto sua publicação estiver sendo considerada pela Revista de Atenção à Saúde.

\section{Referências}

1. Cruz DT et al. Prevalência de Quedas e Fatores Associados em Idosos. Rev Saúde Pública 2012; 46(1): 138-46.

2. Instituto Brasileiro de Geografia e Estatística (IBGE). Transição da estrutura etária no Brasil: oportunidades e desafios para a sociedade nas próximas décadas. Em: Mudança Demográfica no Brasil no Início do Século XXI: Subsídios para as projeções da população. Rio de Janeiro; 2015. Disponível em: <http://biblioteca.ibge. gov.br/visualizacao/ livros/liv93322.pdf $>$. Acesso em: abril/2017.

3. Santos SFC; Silva Neto VM. Treinamento resistido para idosos: revisão de literatura. Cinergis 2017; 18(2): 151-55.

4. Clares JWB, Freitas MC, Borges CL. Fatores sociais e clínicos que causam limitação da mobilidade de idosos. Acta Paulista de Enfermagem 2014; 27(3): 237-42.

5. Gasparotto LPR, Santos JFFQ. A importância da análise dos gêneros para fisioterapeutas: enfoque nas quedas entre idosos. Fisioterapia em Movimento 2012; 25(4): 701-7.

6. Anjos FR et al. Probabilidade de cair e medo de quedas após oficina de equilíbrio em idosos praticantes de atividade física. Rev de Atenção à Saúde 2015; 13(44): 5-10.

7. Oliveira VCS, Furtado F. Instrumentos de avaliação do equilíbrio e mobilidade funcional entre idosos brasileiros ativos sem e com baixo risco para quedas. In: V Simpósio de Pesquisa e Inovação / IV Seminário de Iniciação Científica do IF Sudeste MG - Campus Barbacena; 2014; Barbacena. Minas Gerais: 2014; 1(1).

8. Silva DO et al. Influência da preocupação com quedas na mobilidade e na força de reação do solo em idosas durante descida de escada. Scientia Médica 2014; 24(4): 361-367.

9. Camargos FFO, Dias RC, Dias JMD, Freire MTF. Adaptação transcultural e avaliação das propriedades psicométricas da Falls Efficacy Scale - International em idosos brasileiros (FES-I-BRASIL). Rev Bras Fisioter 2010; 14(3): 237-43.

10. Dutra MC, Cabral ALLC, Azevedo G. Tradução para o português e validação do teste Timed Up and Go. Rev Interfaces 2016; 3(9): 81-88.

11. Abellan van Kan G, Rolland Y, Andrieu S, Bauer J, Beauchet O, Bonnefoy M, et al. Gait speed at usual pace as a predictor of adverse outcomes in community-dwelling older people an International Academy on Nutrition and Aging (IANA) Task Force. J Nutr Health Aging 2009; 13(10): 881-9

12. Martinez BP, Batista AKMS, Ramos IR, Dantas JC, Gomes IB, Forgiarini LA Jr, Camelier FRW, Camelier AA. Viabilidade do teste de velocidade de marcha em idosos hospitalizados. J Bras Pneumol 2016; 42(3): 196-202. 
13. Kuchemann, BA. Envelhecimento populacional, cuidado e cidadania: velhos dilemas e novos desafios. Soc estado 2012; 27(1): 165-80.

14. Lopes BS, Carvalho GA. Histórico de quedas, capacidade funcional e autoeficácia em idosas comunitárias: uma análise comparativa. Rev da Universidade Vale do Rio Verde 2014; 12(1): 304-16.

15. Pinheiro HA, Vilaca, KHC, Carvalho, GA. Postural stability, risk of falls and fear of falling in elderly with diabetic neuropathy who do therapeutic exercises. Fisioter Pesquisa 2014; 21(2): 127-32.

16. Steter AM, Coutinho RMC, Fernandes ACP, Costa LFV. Importância da avaliação do equilíbrio e marcha do idoso. J Health Sci Inst 2014; 32(1):43-7.

17. Ribeiro AS, Santos NKO, Gervásio FM. Atuação da fisioterapia no equilíbrio postural de idosos e sua relação com quedas: revisão estruturada. Rev Movimenta 2014; 7(3): 773-82.

18. Padoin, PG et al. Análise comparativa entre idosos praticantes de exercício físico e sedentários quanto ao risco de quedas. O mundo da saúde 2010; 34(2): 158-64.

19. Alfieri FM, Riberto M, Gatz LS, Ribeiro CPC, Battistella LR. Uso de testes clínicos para verificação do controle postural em idosos saudáveis submetidos a programas de exercícios físicos. Acta Fisiatr 2010; 17(4):153-8.

20. Abreu SSE, Caldas CP. Velocidade de marcha, equilíbrio e idade: um estudo correlacional entre idosas praticantes e idosas não praticantes de um programa de exercícios terapêuticos. Rev Bras de Fisiot 2008; 12(4): 324-30.

21. Nunes NM et al. Avaliação do medo de cair e equilíbrio de idosos ativos e não ativos: um estudo comparativo. Rev bras Ci e Mov 2016; 24(2): 173-81.

\section{Como citar este artigo:}

Müller DVK, Bastos JS. Análise comparativa da mobilidade funcional e medo de quedas de idosas comunitárias. Rev. Aten. Saúde. 2019; 17(62): 05-11. 